\title{
A modified measuring method to investigate the choriocapillaris flow void of polypoidal choroidal vasculopathy with swept source optical coherence tomography angiography
}

\author{
Huajui Wu, Tetsuju Sekiryu, Yukinori Sugano, Kanako Itagaki, Akihito Kasai, Hiroaki Shintake \\ Department of Ophthalmology, Fukushima Medical University, Fukushima City, Fukushima Pref., Japan
}

Contributions: (I) Conception and design: H Wu, T Sekiryu; (II) Administrative support: T Sekiryu; (III) Provision of study materials or patients: T Sekiryu, Y Sugano, A Kasai; (IV) Collection and assembly of data: H Wu, K Itagaki, H Shintake; (V) Data analysis and interpretation: H Wu, T Sekiryu, K Itagaki; (VI) Manuscript writing: All authors; (VII) Final approval of manuscript: All authors.

Correspondence to: Huajui Wu. Department of Ophthalmology, Fukushima Medical University, Fukushima City, Fukushima Pref., Japan. Email: hz210321@gmail.com.

Background: To propose a modified method to investigate the flow void of polypoidal choroidal vasculopathy (PCV) choriocapillaris.

Methods: This paper involves a retrospective study. Included $30 \mathrm{PCV}$ affect eyes, 30 old control eyes, 20 young control eyes, 15 affect eyes with anti-VEGF intravitreal injection treatment, and 8 fellow eyes of anti-VEGF intravitreal injection treatment group. After the choriocapillaris slab [10 $\mu \mathrm{m}$ thick starting $30 \mu \mathrm{m}$ beneath to the retinal pigment epithelium (RPE)-fit reference] was extracted from macular optical coherence tomography angiography $6 \times 6-\mathrm{mm}$ scans, the flow void was segmented by the Phansalkar method. We analyzed the flow void sizes-frequency histogram in order to investigate the differences of flow void proportion between groups. Then we verified the differences between groups after anti-VEGF intravitreal injection treatment.

Results: On the difference curve between the PCV group and Old control group, there was a peak appeared at the flow void sizes range from 900 to $1,125 \mu^{2}$. The average number of flow void sizes from 900 to $1,125 \mu^{2}$ was significantly higher in the Old control group than that in the Young control group $(\mathrm{P}<0.05)$ and there was no difference between the affect eyes group and the Old control group. The proportion of flow void sizes from 900 to $1,125 \mu^{2}$ were remarkably higher in the affect eyes group compared to the Old control group $(\mathrm{P}<0.05)$, showing no difference between the Young control group and the Old control group. The average number of flow void sizes from 900 to $1,125 \mu^{2}$ and the proportion of flow void sizes range from 900 to $1,125 \mu^{2}$ were significantly higher in the treatment group after the treatment $(\mathrm{P}<0.05)$ and there was no difference in the fellow eyes of treatment group. The choroidal thickness was significantly reduced after the treatment of the treatment group $(\mathrm{P}<0.001)$, while the fellow eyes of the treatment group had no difference.

Conclusions: Our method was specific for the pathological changes in choriocapillaris structures of PCV affect eyes, fellow eyes, and the affect eyes after anti-VEGF treatment.

Keywords: Choriocapillaris (CC); flow void; polypoidal choroidal vasculopathy (PCV); optical coherence tomography angiography (OCTA)

Submitted Sep 02, 2020. Accepted for publication Mar 23, 2021.

doi: $10.21037 /$ qims-20-1027

View this article at: http://dx.doi.org/10.21037/qims-20-1027 


\section{Introduction}

Optical coherence tomography angiography (OCTA) is a fast and non-invasive technology in recent years. The basic principle is to perform multiple scans in the same location. The changes of the reflex signal during each scan indicates that there is a moving object. By measuring the changes of the signals in the vessel cavity and merge the images, we get a complete retinal choroidal blood vessel image (1). Due to the physical limitations of laser sources, OCTA has limits in resolution. In this research, we used Plex Elite 9000, swept-source OCTA (SS-OCTA) (Carl Zeiss Meditec Inc., Dublin, California, USA). The axial resolution of SSOCTA is $6.3 \mu \mathrm{m}$, and lateral resolution $(\mathrm{Lr})$ is $20 \mu \mathrm{m}$ on retina surface.

Choriocapillaris (CC) is the innermost layer of the choroidal vasculature. It is composed of a dense capillary network that provides the oxygen and nutrients for the retinal pigment epithelium (RPE) and the outer layer of the retina.

Flow void (FV) of $\mathrm{CC}$ is an alternative way to measure the CC structure. The standard vessel diameter of CC is $16-20 \mu \mathrm{m}(2)$, smaller than the $\mathrm{Lr}$ of the SS-OCTA instrument. Therefore, some intracapillary distances which are bigger than the Lr can be measured to represent the CC structure where the areas without blood perfusion.

Polypoidal choroidal vasculopathy (PCV) was first proposed by Yannuzzi. Clinical manifestation is the branching vascular network with polypoid lesion structure (3), generally diagnosed through indocyanine green angiography. Recent research indicates that OCTA can detect branching vascular network and polypoidal components more accurately than indocyanine green angiography (4).

The anti-VEGF intravitreal injection (AVI) has been approved in many countries for years and has shown promising safety and effectiveness. Previous studies have shown that after the AVI treatment, choroidal thickness (CT) was reduced in pachychoroid neovasculopathy (PNV), myopic choroidal neovascularization (CNV), and neovascular age-related macular degeneration (nAMD) (5-7).

In the previous FV researches, there were several problems under the premise of the fixed depth of the CC slab: (I) there were lots of binarization methods in previous researches, whose results were different; (II) for the auto local threshold methods, different radius selections led to different results (8-14); (III) previous investigations did not take the physical limitations of OCTA instruments into account; (IV) used wrong statistics (average and standard deviation) to assess the FV $(15,16)$. To solve the previous problems, we proposed a modified method in this research.

We assumed that since PCV is often accompanied by the abnormality of CC structure (17-19), the morphology of the CC may change after PCV.

The main goal of this research is to propose a method to evaluate the non-normal distribution of FV.

\section{Methods}

\section{Research design}

The authors are accountable for all aspects of the work in ensuring that questions related to the accuracy or integrity of any part of the work are appropriately investigated and resolved. The study was conducted in accordance with the Declaration of Helsinki (as revised in 2013). This retrospective study was approved by the Institutional Review Board of Fukushima Medical University of 2020091 and individual consent for this retrospective analysis was waived.

This research is inclusive of two parts. First, the FV sizes-frequency histogram analysis. In this part, we compared the distributions of total FV and all Early Treatment of Diabetic Retinopathy Study (ETDRS) subfields of the PCV group, Old control group (age-matched normal control), and Young control group to determine the range of FV. Second, the FV validation part. We discussed the differences before and after AVI treatment in the AVI group and fellow eyes of the AVI group. We also verified the differences in the subfields without pathological changes of the AVI group.

\section{Participants}

One hundred and three eyes from 85 Japanese were included in this study in total, including 6 groups: PCV group: 30 eyes of 30 patients diagnosed with unilateral PCV at the Department of Ophthalmology of Fukushima Medical University Hospital, Fukushima City, Japan. Old control group (age-matched control group): 30 fellow eyes of 30 patients diagnosed with retinal vein occlusion (RVO). Young control group: 20 eyes of 10 regular young volunteers. AVI group: another 15 treatment-naive eyes of PCV patients who received three AVI treatments and two tests before and after treatment. AVI-fellow group: 8 fellow eyes of AVI group without any observable pathological 
changes on the B-scan of SS-OCT and received two tests with 3-month intervals. AVI normal group: the ETDRS grid subfields without any detectable pathological changes on the B-scan of SS-OCT from the AVI group.

Exclusion criteria were: (I) the eyes with any combined retinal diseases, such as diabetic retinopathy (DR), glaucoma, and central serous chorioretinopathy (CSC); (II) the refractive error was greater than -6 diopters; (III) the patients with any systemic diseases, such as hypertension or diabetes; (IV) any segmentation errors were on the B-scan of SS-OCT images.

\section{Clinical examination}

The examinations included indirect ophthalmoscopy, slit-lamp biomicroscopy, and color and red-free fundus photography. The CT was manually measured by a masked ophthalmologist (Y Sugano) three times and took the average as the vertical distance between the RPE and the choroidoscleral border at the center of the fovea, using the B-scan of SS-OCT through ImageJ software, version 1.52p (National Institutes of Health, Bethesda, Maryland, USA).

\section{Imaging}

Subjects underwent SS-OCTA imaging using the PLEX Elite 9000 device, which uses a swept laser source with a central wavelength of $1,050 \mathrm{~nm}$. The device operates at $100,000 \mathrm{~A}$-scans per second, and $\mathrm{Lr}$ is $20 \mu \mathrm{m}$. OCTA image included a $6 \times 6 \mathrm{~mm}$ area centered on the macula. Signal strength index $>7$ was defined as sufficient image quality. A fully-automated, build-in retinal layer segmentation was applied to segment the CC slab $(10 \mu \mathrm{m}$ thick starting $30 \mu \mathrm{m}$ beneath to the RPE-fit reference) $(20,21)$.

\section{Image enhancement}

We imported the angiography image of the $\mathrm{CC}$ and the correspondent en face structural image into MATLAB R2019a. Because both images were in the different sizes, we first used the build-in function "imresize" to extend them into $1,200 \times 1,200$ pixels $(5 \mu \mathrm{m} /$ pixel). Then we used the function "mat2gray" to make normalized image. We modified the compensation algorithm published before for the signal to eliminate the influence of the shadowing effect on the quantitation of the CC (22).

$$
\text { Fcompensated }=\operatorname{Aim} \times(1-\operatorname{Norm}(\operatorname{Sim}))
$$

Where Norm () represents the normalized image, Sim is the structural OCT image, and Aim is the OCTA image. We used "fspecial" to apply Gaussian filter (hsize $=5$ pixels, sigma $=0.5$, "replicate" for the boundary). The filter size should be bigger than Lr.

\section{Image binarization}

We chose the Phansalkar method (23) that specifically increases the threshold in the low contrast areas as the binarization algorithm in this research. We imported enhanced images into ImageJ software and processed "Auto Local Threshold" with the Phansalkar method for thresholding (radius $=5$ pixels, $\mathrm{k}=0.25, \mathrm{r}=0.5$ ). The local average and standard deviation of the Phansalkar method in ImageJ was defined as the results of all pixels within the circle with "radius". In this circle, it should not include both normal contrast areas and low contrast areas to prevent all low contrast areas from mistakenly being the same category. Thus the "radius" should be smaller than the shadows caused by blood vessels and other objects and bigger than the Lr. After thresholding, all signals with a diameter smaller than the Lr were unreliable and ought to be excluded. Therefore, we applied "Analyze Particles" to eliminate the FV sizes which the diameters were smaller than Lr. Then, we used "Watershed Irregular Features" (24) to separate the connected FV, whose junctions were smaller than Lr.

\section{ETDRS grid}

After binarization, we imported the $\mathrm{FV}$ images into MATLAB to establish the ETDRS grid. We used "InsertShape" (LineWidth =1, Color =Black) to draw ETDRS grid on the images. We separated the FV images into 11 subfields: $\mathrm{C} 1: 1-\mathrm{mm}$ circle at the center of macula, C2: 3-mm rim, C3: 6-mm rim, inner superior, inner nasal, inner inferior, inner temporal, outer superior, outer nasal, outer inferior, outer temporal.

\section{FV sizes-frequency histogram analysis}

We used "Analyze Particles" of Image to calculate the FV in 11 subfields, sizes range from 0 to 1,000 pixels $\left(25,000 \mathrm{\mu m}^{2}\right)$. We imported FV sizes data into Excel (Microsoft Office 2019; Microsoft Inc., Redmond, Washington, USA) and used the built-in function "Frequency" with 10 pixels $\left(250 \mathrm{~m}^{2}\right)$ intervals from 16 pixels $\left(400 \mu \mathrm{m}^{2}\right)$ to 1,000 pixels 
$\left(25,000 \mu \mathrm{m}^{2}\right)$. To analyze the sizes-FV\% histogram of the Old control group, Young control group and PCV group in each sizes interval.

The FV\% (the proportion of each FV interval) was defined as the ratio of $\mathrm{FV}$ number in each interval to total FV.

$$
F V \%=\frac{\text { Number of } F V \text { of eachinterval }}{\text { Total } F V \times \text { number of eyes }} \times 100 \%
$$

Then, we minus the FV\% of the Young control group from the Old control group to detect the differences between the normal subjects with different ages. As well as the Old control group from the PCV group to detect the differences between same age subjects with or without PCV. We plotted the results into FV sizes-log FV\% and the differences of $\mathrm{FV} \%$ chart.

The $\mathrm{FV}_{1125}$ was defined as the average number of the $\mathrm{FV}$, sizes from 36 to 45 pixels (900 to $1,125 \mu^{2}$ ).

The $\mathrm{FV}_{1125} \%$ (the proportion of $\mathrm{FV}_{1125}$ ) was defined as the ratio of the number of $\mathrm{FV}_{1125}$ to total number of $\mathrm{FV}$.

$$
F V_{1125} \%=\frac{\text { Number of } F V_{1125}}{\text { Total number of } F V} \times 100 \%
$$

The AVI normal group were defined as the normal subfields based on the ETDRS grid, which we compared the FV result images with the SS-OCT images of AVI group.

\section{FV validation}

We compared the FV distribution of PCV eyes and healthy eyes in different-age groups (PCV group, Old control group, and Young control group) to confirm whether $\mathrm{FV}_{1125}$ and $\mathrm{FV}_{1125} \%$ had the age correlation as the previous method.

We compared the $\mathrm{FV}_{1125}$ changes of AVI group before and after three AVI treatments, the AVI-fellow group with 3-month interval, to confirm whether there was a difference after AVI treatment.

\section{Statistical analysis}

All variables were reported as average and standard deviation. The Kolmogorov-Smirnov test was performed to detect the normality distribution of FV. One-way analysis of variance (one-way ANOVA) with Tukey post hoc test has calculated the differences between PCV group, Old control group, and Young control group. The paired Student's t-test was conducted to investigate the differences between AVI group and AVI-fellow group. Intraclass correlation coefficients (ICC) were calculated for the agreement for the FV among AVI group and AVI-fellow group with 3-month interval. Pearson's correlation was performed to evaluate the correlation between age and FV, and the changes of CT and FV. Statistical calculations were performed using SPSS Statistics V.26 (IBM Corporation, Armonk, NY, USA). The statistical significance level was $\mathrm{P}<0.05$.

\section{Results}

\section{Baseline characteristics}

Average age was: $70.6 \pm 7.2$ years (55-86 years) in PCV group and $70.9 \pm 6.8$ years ( $58-88$ years) in the Old control group, 25.2 \pm 2.6 years (21-29 years) in the Young control group, $74.5 \pm 4.7$ years (63-85 years) in AVI group. The demographic characteristics of each group are shown in Table 1.

\section{FV sizes-frequency histogram analysis}

In the histogram analysis, there was a peak at FV sizes from 36 to 45 pixels $\left(900-1,125 \mu^{2}\right)$ on the curve of the differences between PCV group and Old control group. There was another peak between the Old control group and the Young control group (Figure 1A). Furthermore, we analyzed 11 subfields according to the ETDRS grids between the PCV group and the Old control group, and we found that there were also similar peaks in all subfields (Figure 1B).

We summarized the sizes and spacing of the FV sizes with the most frequently appearing peaks in all subfields: The highest frequency of sizes was from 36 to 45 pixels (900$1,125 \mu^{2}$ ), which were subfields C1, C3, inner nasal, outer superior, outer nasal, outer inferior, and outer temporal, 7 of 11 subfields (63.6\%). The second was from 46 to 55 pixels $\left(1,150-1,375 \mu^{2}\right)$, which were subfields $C 2$, inner superior, and inner temporal, 3 of 11 subfields ( $27.3 \%)$. The third was from 56 to 65 pixels $\left(1,400-1,625 \mu^{2}\right)$, which was subfield inner inferior, 1 of 11 subfields (9.1\%) (Figure $1 B$ ). Therefore, we found the interval of $900-1,125 \mu^{2}$ was the FV sizes related to the pathological changes of PCV.

\section{Validation of the normal distribution}

The Kolmogorov-Smirnov test results of $\mathrm{FV}_{1125} \%$ of the 
Table 1 Characteristics of participant groups (average \pm SD)

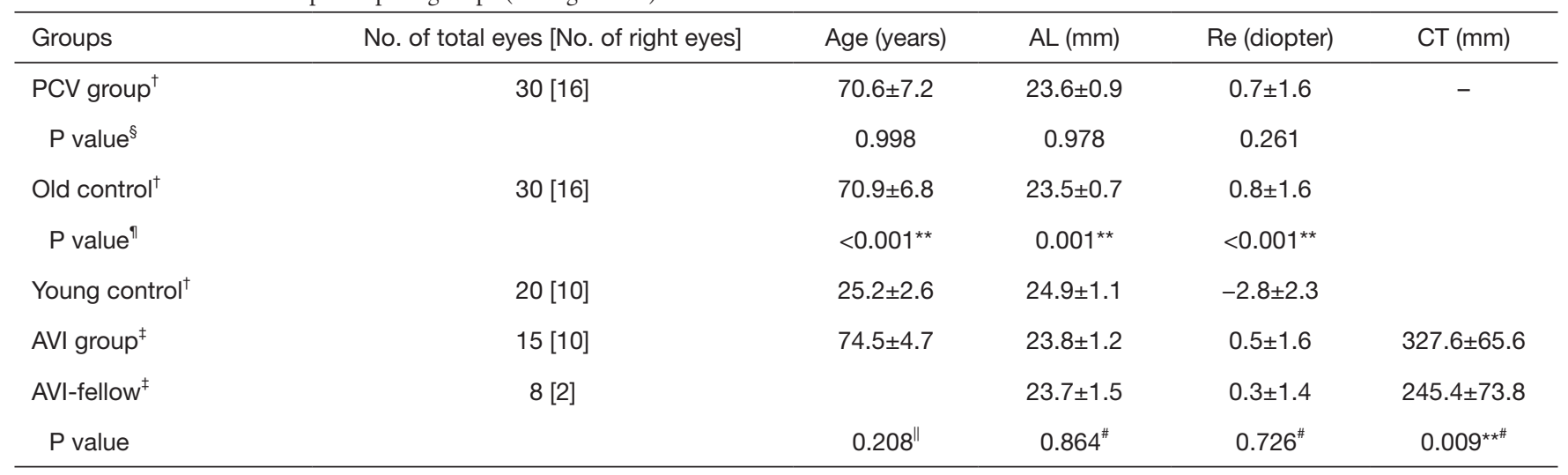

${ }^{\dagger}$, one-way ANOVA with Tukey post-hoc test; ${ }^{\ddagger}$, Student's $t$-test; ${ }^{\S}, \mathrm{P}$ value of PCV group-Old control group; ^, P value of Old control group-Young control group; ", P value of AVI group-PCV group; ", P value of AVI group-AVI-fellow group; ${ }^{\star}, \mathrm{P}<0.01$. PCV group: PCV affect eye group; Old control: age-matched control group; AVI group: anti-VEGF intravitreal injection group; AVI-fellow group: fellow eyes of AVI group. CT, choroidal thickness; AL, axial length; Re, refractive error; PCV, polypoidal choroidal vasculopathy; AVI, anti-VEGF intravitreal injection; SD, standard deviation.
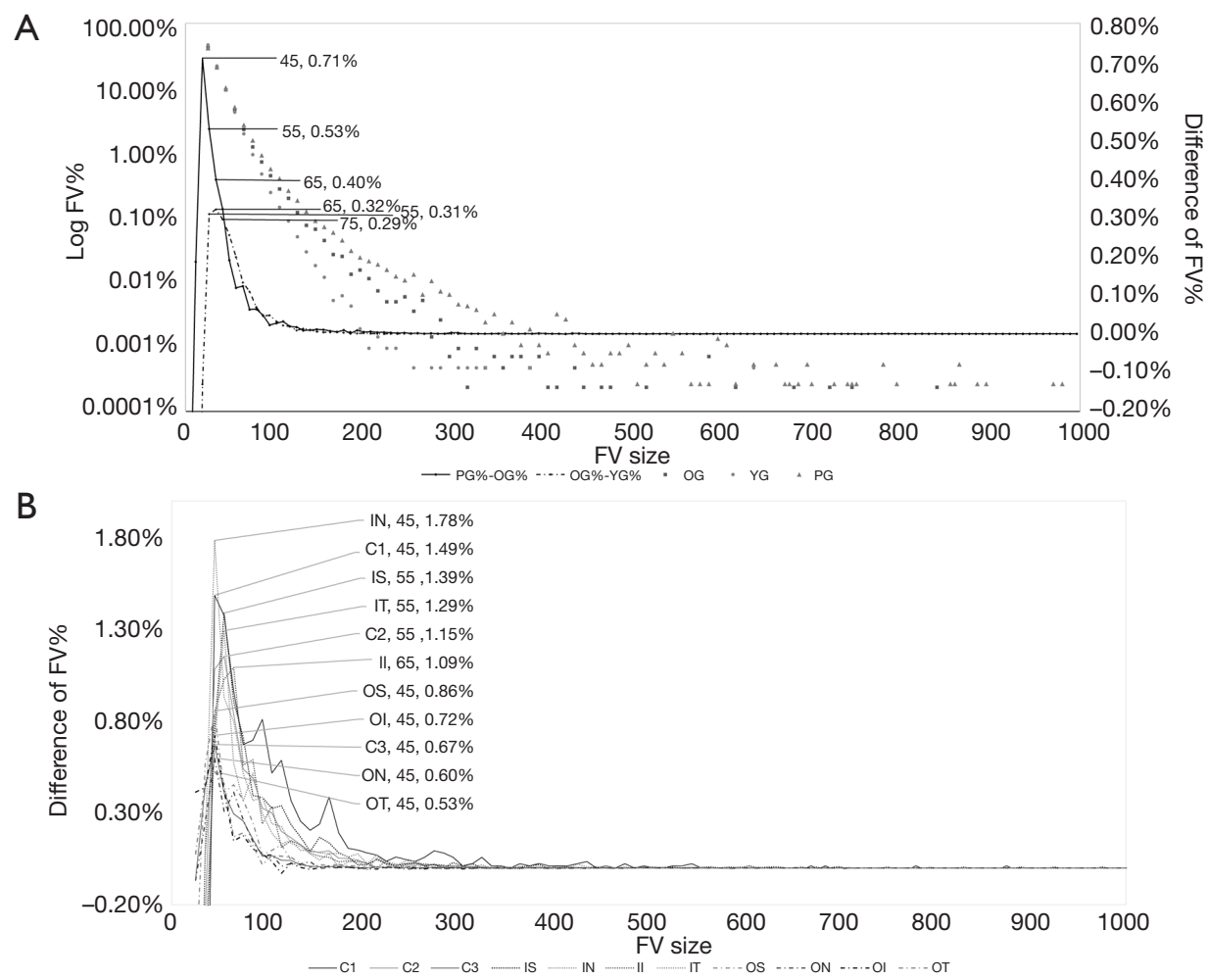

Figure 1 The characteristic of the FV\% distribution. (A) The sizes-log FV\% and difference of FV\% histogram between PCV group, Old control group, and Young control group. The differences of total FV\% shows a peak among disease groups (PCV group- Old control group) and another peak among normal aging groups (Old control group-Young control group). (B) The differences of FV\% histogram between PCV group and Old control group in 11 subfields. All subfields showed the similar peaks. OG: age-matched control group; YG: Young control group; PG: PCV affect eye group; C1: 1-mm circle at the center of macula; C2: 3-mm rim; C3: 6-mm rim; 45 : FV sizes from 36 to 45 pixels $\left(900-1,125 \mu^{2}\right)$; 55: FV sizes from 46 to 55 pixels $\left(1,150-1,375 \mu^{2}\right) ; 65$ : FV sizes from 56 to 65 pixels $\left(1,400-1,625 \mu \mathrm{m}^{2}\right)$. IS, inner superior; IN, inner nasal; II, inner inferior; IT, inner temporal; OS, outer superior; ON, outer nasal; OI, outer inferior; OT, outer temporal. 
Table 2 Validation of the flow void with age dependency ${ }^{\dagger}$ (average \pm SD)

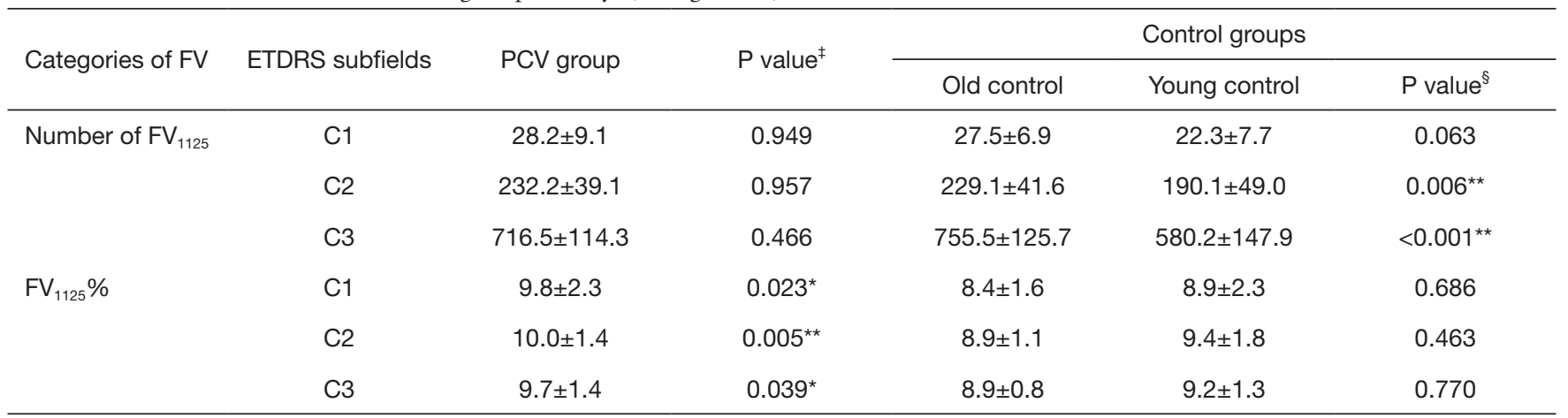

${ }^{\dagger}$, one-way ANOVA with Tukey post-hoc test; ${ }^{\ddagger}, \mathrm{P}$ value of PCV group-Old control group; ${ }^{\S}$, P value of Old control group-Young control group; *, $\mathrm{P}<0.05 ;{ }^{* *}, \mathrm{P}<0.01$. Number of $\mathrm{FV}_{1125}$ : average number of the $\mathrm{FV}$ size from 900 to $1,125 \mu \mathrm{m}^{2} ; \mathrm{FV}_{1125} \%$ : Proportion of $\mathrm{FV}_{1125} ; \mathrm{PCV}$ group: PCV affect eye group; Old control: age-matched control group; C1: 1-mm circle at the center of the macula; C2: 3-mm rim; C3: 6-mm rim. PCV, polypoidal choroidal vasculopathy; SD, standard deviation; FV, flow void.

PCV group, Old control group, and Young control group were normal distribution $(\mathrm{C} 1: \mathrm{P}=0.200, \mathrm{C} 2: \mathrm{P}=0.200, \mathrm{C} 3$ : $\mathrm{P}=0.082$ ).

\section{Validation of the FV with age dependency}

In the comparison of PCV group, Old control group and Young control group, the $\mathrm{FV}_{1125}$ were positively correlated with ages in $\mathrm{C} 1(\beta=0.265, \mathrm{P}=0.017), \mathrm{C} 2(\beta=0.369, \mathrm{P}=0.001)$, and $\mathrm{C} 3(\beta=0.445, \mathrm{P}<0.001)$.

The average number of $\mathrm{FV}_{1125}$ were significantly higher in the Old control group compared to the Young control group in $\mathrm{C} 2(\mathrm{P}=0.006)$ and $\mathrm{C} 3(\mathrm{P}<0.001)$. There were no significant differences among the $\mathrm{PCV}$ group and the Old control group in $\mathrm{C} 1, \mathrm{C} 2$ and $\mathrm{C} 3(\mathrm{P}>0.05)$ (Table 2, Figure 2A).

The $\mathrm{FV}_{1125} \%$ were significantly higher in the PCV group compared to the Old control group in $\mathrm{C} 1(\mathrm{P}=0.023)$, C2 $(\mathrm{P}=0.005)$ and $\mathrm{C} 3(\mathrm{P}=0.039)$. There were no significant differences among the Old control group and the Young control group in $\mathrm{C} 1, \mathrm{C} 2$ and $\mathrm{C} 3(\mathrm{P}>0.05)$ (Figure $2 B)$.

\section{The FV changes after AVI treatment}

The average number of $\mathrm{FV}_{1125}$ had an increasing trend after the treatment in the AVI group. Among them, C2 $(+18.9 \pm 33.4, \mathrm{P}=0.045)$ and $\mathrm{C} 3(+78.3 \pm 71.4, \mathrm{P}=0.001)$ reached statistical significance. Also significantly increased in the AVI normal group $(+20.2 \pm 21.9, \mathrm{P}=0.001)$. Compared to the AVI-fellow group, the changes in $\mathrm{C} 1, \mathrm{C} 2$, and $\mathrm{C} 3$ had no statistical differences after treatments $(\mathrm{P}>0.05)$
(Figure $3 A$ ). $\mathrm{FV}_{1125} \%$ had an increasing trend in the AVI group. Among them, $\mathrm{C} 3(+0.4 \% \pm 0.6 \%, \mathrm{P}=0.022)$ reached statistical significance. And significantly increased in the AVI normal group $(+0.6 \% \pm 0.9 \%, \mathrm{P}=0.019)$. Compared to the AVI-fellow group, changes in $\mathrm{C} 1, \mathrm{C} 2$, and $\mathrm{C} 3$ had no statistical differences after treatments $(\mathrm{P}>0.05)$ (Figure $3 B)$.

\section{The CT changes after AVI treatment}

Compared to the baseline, the average CT of AVI group after treatment was $222.5 \pm 46.8 \mu \mathrm{m}$, significantly decreased after treatment $(-105.1 \pm 66.4 \mu \mathrm{m}, \mathrm{P}<0.001)$, while the CT of AVI-fellow group after 3-month interval was 231.6 21.5 $\mu \mathrm{m}$, was not statistically significant $(-13.8 \pm 25.7 \mu \mathrm{m}$, $\mathrm{P}=0.145$ ).

The differences after treatment between AVI group and AVI-fellow group was $-27.9 \pm 79.7 \mu \mathrm{m}$, and had no statistical difference $(\mathrm{P}=0.720)$.

\section{Reproducibility}

The ICC of 22 subfields of $\mathrm{FV}_{1125}$ (before and after AVI treatment) was 0.913 (95\% CI: 0.853-0.956).

\section{Discussion}

In this retrospective study, we found that the number and the proportion of $\mathrm{FV}$ sizes from 900 to $1,125 \mu^{2}$ significantly higher in PCV eyes compared to the healthy elderly. The differences also occurred after AVI treatment.

The histogram of $\mathrm{FV}$ in each group was similar 

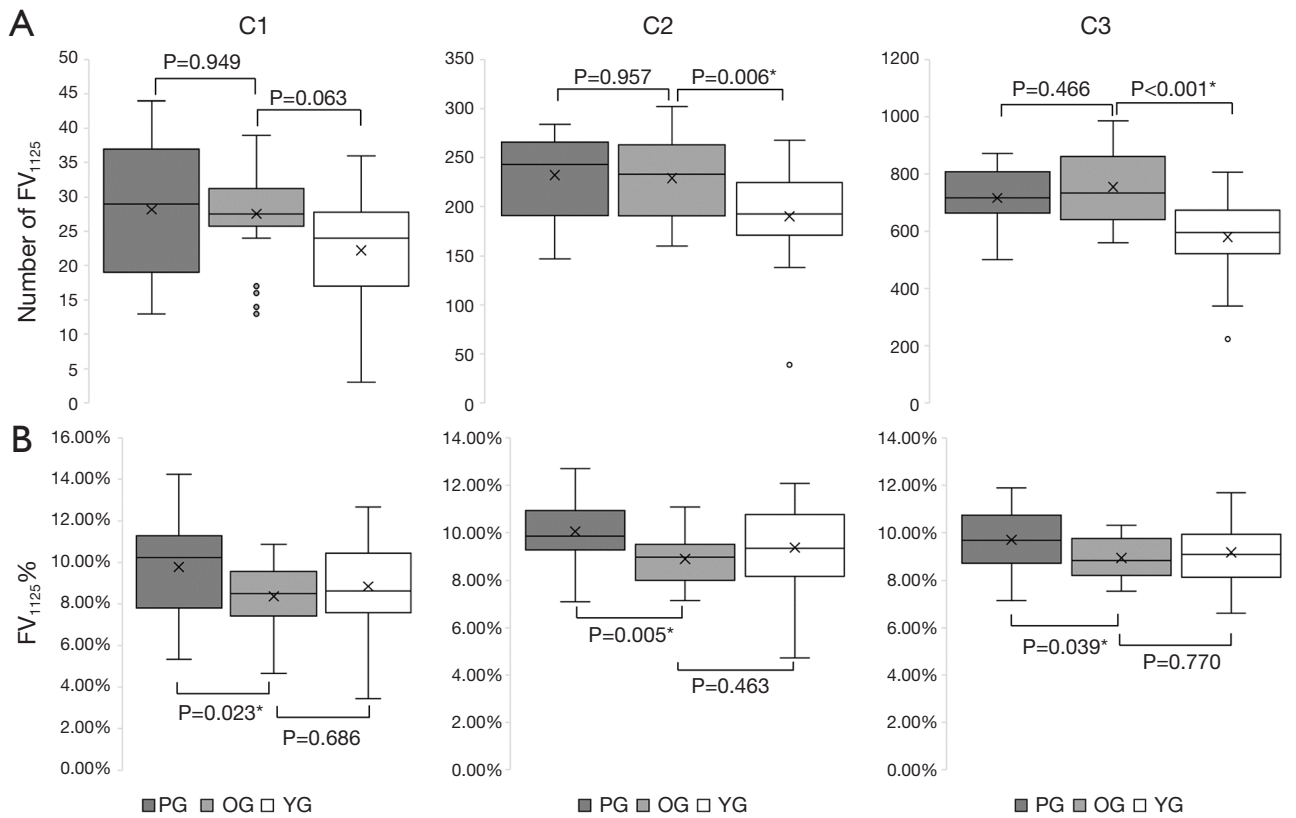

Figure 2 The comparison of $\mathrm{FV}_{1125}$ and $\mathrm{FV}_{1125} \%$ between PCV group and normal control groups. (A) The average number of $\mathrm{FV}$ 1125 were significantly higher in the Old control group compared to the Young control group in C2 and C3, and there were no differences among PCV group and Old control group in all subfields. (B) The $\mathrm{FV}_{1125} \%$ were significantly higher in PCV group compared to Old control group in all subfields, and there were no differences among normal control groups. *, P<0.05. PG: PCV affect eye group; OG: Age-matched control group;

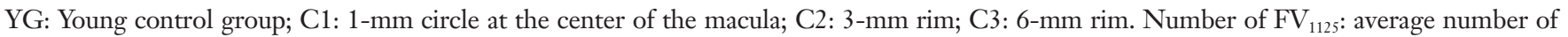
the FV sizes from 900 to $1,125 \mu^{2} ; \mathrm{FV}_{1125} \%$ : proportion of $\mathrm{FV}_{1125}$.

to the results of Spaide and followed the power-law distribution (25). The sizes of the FV we proposed is different from the results of Borrelli et al. for intermediate age-related macular degeneration (iAMD) $(10,26)$. Although there is no relevant research published for the $\mathrm{FV}$ characteristics of $\mathrm{PCV}$, considering the characteristics of the power-law distribution, the statistics used for the normal distribution (average and standard deviation) are inapplicable $(15,16)$. Moreover, we prove that once we limited the observed sizes to a specific range (e.g., 900 to $1,125 \mathrm{\mu m}^{2}$ ), the FV were normally distributed and suitable for those statistics.

The $\mathrm{FV}_{1125}$ was positively correlated with age, which is consistent with the previous research (27). In the comparison between healthy groups (Old control group and Young control group), the $\mathrm{FV}_{1125}$ is significantly higher in the Old control group, and there is no difference between aging eyes (PCV group and Old control group), indicating the change of the $\mathrm{FV}_{1125}$ was related to the increasing of the total number of $\mathrm{FV}$ by aging.

The $\mathrm{FV}_{1125} \%$ is significantly higher in the PCV eyes compared to the normal aging eyes (PCV group and Old control group), and it shows no difference between healthy groups (Old control group and Young control group), indicated $\mathrm{FV}_{1125} \%$ was related to the pathological changes of CC.

Our method removed the binary results which were smaller than the Lr before analysis to prevent a large number of unreliable noises from distorting the analysis results. (Figure 4). The previous studies have shown that the differences between the disease and the control group was small $(10,22,28,29)$, the noise could have a direct impact on the analysis.

After we removed the noise (Figure $4 A$ ), we found several small areas without FV. This was due to normal CC structures, whose intracapillary distances were smaller than $\operatorname{Lr}(2)$, and the SS-OCTA cannot discriminate.

The $\mathrm{FV}_{1125}$ was significantly higher, and the $\mathrm{CT}$ was significantly lower in the AVI normal group. And had no difference between the AVI normal and the AVI-fellow group after treatment. Indicated that the increasing of the $\mathrm{FV}_{1125}$ was related to the increased total number of $\mathrm{FV}$, 
A

C
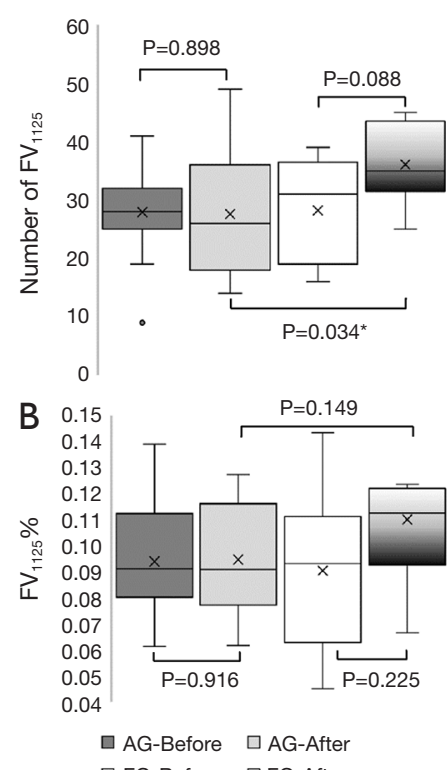

$\square$ FG-Before $\quad \square$ FG-After
C2
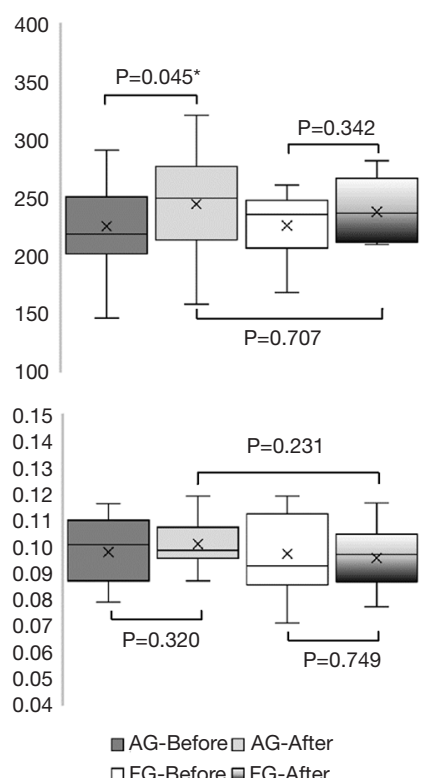

$\mathrm{C} 3$

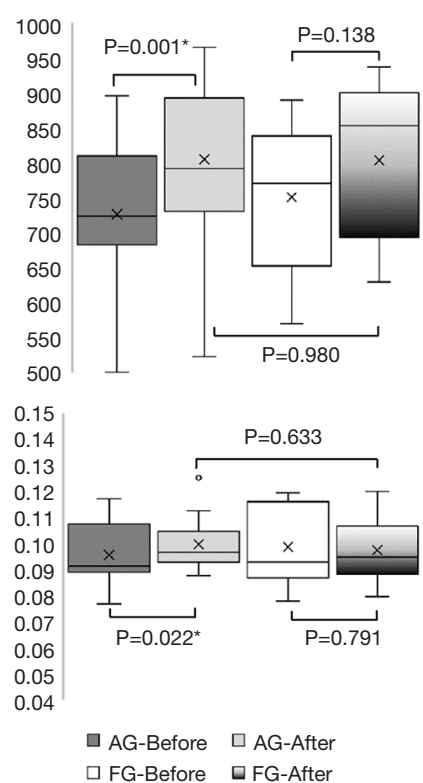

AGn

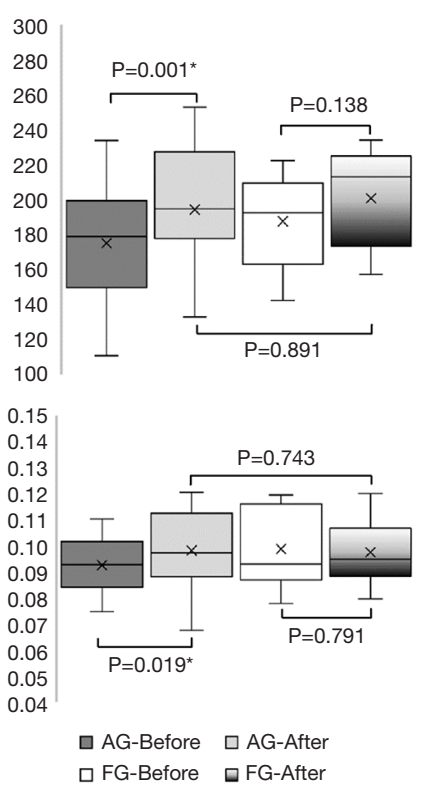

Figure 3 The $\mathrm{FV}_{1125}$ and $\mathrm{FV}_{1125} \%$ of AVI group and AVI-fellow group before and after treatment. (A) The average number of $\mathrm{FV}$ 1125 were significantly higher in C2 and C3 and AGn after anti-VEGF treatment, and there were no differences among AVI-fellow groups. (B) The $\mathrm{FV}_{1125} \%$ had an increasing trend after anti-VEGF treatment. Among them, C3 and AGn reached statistical significance, and there were no differences among AVI-fellow groups. * $\mathrm{P}<0.05$. AG-Before: anti-VEGF intravitreal injection group before treatment; AG-After: anti-VEGF intravitreal injection group after three times treatment; FG-Before: fellow eyes of AVI group before 3 months interval; FG-After: fellow eyes of AVI group after 3 months interval; C1: 1-mm circle at the center of the macula; C2: 3-mm rim; C3: 6-mm rim; AGn: subfields without pathological changes of AVI group; Number of $\mathrm{FV}_{1125}$ : average number of the $\mathrm{FV}$ sizes from 900 to $1,125 \mu^{2} ; \mathrm{FV}_{1125} \%$ : proportion of $\mathrm{FV}_{1125}$. FV: flow void; AVI, anti-VEGF intravitreal injection.

which was due to the anti-VEGF agent effects, caused the reduction of CT and CC diameter (30-32). The $\mathrm{FV}_{1125} \%$ of AVI normal group significantly raised to the AVI-fellow group level, indicated that the increasing of $\mathrm{FV}_{1125} \%$ was not only due to the CT change, but both PCV affect eyes and fellow eyes had similar pathological changes of CC $(33,34)$, and we provided a possible way to detect those pathological changes.

The CT significantly decreased after treatment was consistent with previous researches $(35,36)$. We expected that the CT change was negatively correlated with the FV change of the AVI normal group. But the result was not statistically significant $(\beta=-0.336, P=0.343)$. We thought it was related to the histological position of AVI normal group. Since 17 images of AVI normal group belongs to the C3 subfields (94.4\%) and 1 image belongs to the C2 subfield (5.6\%). The location of the CT measurement was at the center of $\mathrm{C} 1$, which may cause some impacts. Our results of the CT and the FV relationship of the fellow eye were consistent with the previous results (5-7). It indicated that our results were specific for pathological changes in the CC structure.

The limitations are the small sample size, especially the small number of the AVI normal group, and the limited exploration of relatively normal subfields of CC in PCV; Our result was a relatively narrow interval, and the conclusion will be different because of the different peak range selection and the $\mathrm{Lr}$ of the different instruments; the FV sizes range should be different with different diseases because the various pathological changes in the $\mathrm{CC}$ structure.

\section{Conclusions}

In this research, we proposed a modified method to invest the FV which followed a non-normal distribution. The proportion of sizes interval (e.g., $\mathrm{FV}_{1125} \%$ ) was specific for pathological changes in the CC structure. The CC perfusion and CT of PCV significantly decreased after AVI 

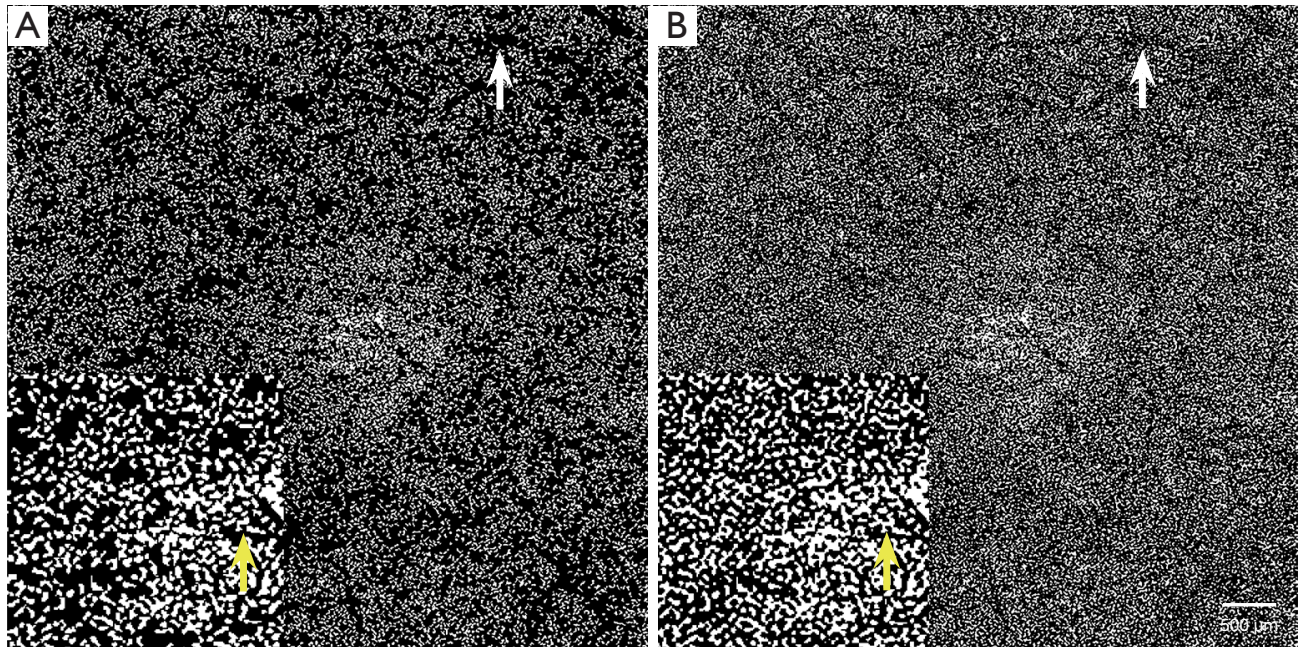

Figure $4 \mathrm{~A}$ PCV affect eye from 74 years old male demonstrate how Lr affect the FV results. (A) Our method removed the noise smaller than $\operatorname{Lr}^{2}\left(20 \times 20 \mu \mathrm{m}^{2}\right)$. The total FV area is $22.8 \%$. (B) The same subject with small noise. The total FV area is $29.2 \%$. White arrow: (A) our method removes the noise and reveals the shadow of vessels; (B) a lot of small noise can be seen in the shadow of superficial vessels. Composition: $1 \mathrm{~mm}^{2}$ centered at the macula. Yellow arrow: (A) our method divides the FV into reasonable sizes after removed the noise; (B) the FV incorrectly connected into clusters. FV, flow void; PCV, polypoidal choroidal vasculopathy; Lr, lateral resolution.

treatment.

\section{Acknowledgments}

Funding: None.

\section{Footnote}

Conflicts of Interest: All authors have completed the ICMJE uniform disclosure form (available at http://dx.doi. org/10.21037/qims-20-1027). The authors have no conflicts of interest to declare.

Ethical Statement: The authors are accountable for all aspects of the work in ensuring that questions related to the accuracy or integrity of any part of the work are appropriately investigated and resolved. The study was conducted in accordance with the Declaration of Helsinki (as revised in 2013). This retrospective study was approved by the Institutional Review Board of Fukushima Medical University of 2020-091 and individual consent for this retrospective analysis was waived.

Open Access Statement: This is an Open Access article distributed in accordance with the Creative Commons Attribution-NonCommercial-NoDerivs 4.0 International
License (CC BY-NC-ND 4.0), which permits the noncommercial replication and distribution of the article with the strict proviso that no changes or edits are made and the original work is properly cited (including links to both the formal publication through the relevant DOI and the license). See: https://creativecommons.org/licenses/by-nc-nd/4.0/.

\section{References}

1. Kashani AH, Chen CL, Gahm JK, Zheng F, Richter GM, Rosenfeld PJ, Shi Y, Wang RK. Optical coherence tomography angiography: A comprehensive review of current methods and clinical applications. Prog Retin Eye Res 2017;60:66-100.

2. Olver JM. Functional anatomy of the choroidal circulation: methyl methacrylate casting of human choroid. Eye (Lond) 1990;4:262-72.

3. Yannuzzi LA, Sorenson J, Spaide RF, Lipson B. Idiopathic polypoidal choroidal vasculopathy (IPCV). Retina 1990;10:1-8.

4. Kim K, Yang J, Feuer W, Gregori G, Kim ES, Rosenfeld PJ, Yu SY. A Comparison Study of Polypoidal Choroidal Vasculopathy Imaged with Indocyanine Green Angiography and Swept-Source Optical Coherence Tomography Angiography. Am J Ophthalmol 2020;217:240-51. 
5. Azuma K, Tan X, Asano S, Shimizu K, Ogawa A, Inoue T, Murata H, Asaoka R, Obata R. The association of choroidal structure and its response to anti-VEGF treatment with the short-time outcome in pachychoroid neovasculopathy. PLoS One 2019;14:e0212055.

6. Ng WY, Ting DS, Agrawal R, Khandelwal N, Htoon HM, Lee SY, Wong TY, Cheung GC. Choroidal Structural Changes in Myopic Choroidal Neovascularization After Treatment with Antivascular Endothelial Growth Factor Over 1 Year. Invest Ophthalmol Vis Sci 2016;57:4933-9.

7. Inan S, Baysal Z, Inan UU. Long-Term Changes in Submacular Choroidal Thickness after Intravitreal Ranibizumab Therapy for Neovascular Age-Related Macular Degeneration: 14-Mo Follow-Up. Curr Eye Res 2019;44:908-15.

8. Borrelli E, Souied EH, Freund KB, Querques G, Miere A, Gal-Or O, Sacconi R, Sadda SR, Sarraf D. Reduced choriocapillaris flow in eyes with type 3 neovascularization and age-related macular degeneration. Retina 2018;38:1968-76.

9. Uji A, Balasubramanian S, Lei J, Baghdasaryan E, AlSheikh M, Sadda SR. Choriocapillaris Imaging Using Multiple En Face Optical Coherence Tomography Angiography Image Averaging. JAMA Ophthalmol 2017;135:1197-204.

10. Borrelli E, Shi Y, Uji A, Balasubramanian S, Nassisi M, Sarraf D, Sadda SR. Topographic Analysis of the Choriocapillaris in Intermediate Age-related Macular Degeneration. Am J Ophthalmol 2018;196:34-43.

11. Nassisi M, Shi Y, Fan W, Borrelli E, Uji A, Ip MS, Sadda SR. Choriocapillaris impairment around the atrophic lesions in patients with geographic atrophy: a swept-source optical coherence tomography angiography study. Br J Ophthalmol 2019;103:911-7.

12. Nassisi M, Baghdasaryan E, Tepelus T, Asanad S, Borrelli E, Sadda SR. Topographic distribution of choriocapillaris flow deficits in healthy eyes. PLoS One 2018;13:e0207638.

13. Spaide RF. Choriocapillaris signal voids in maternally inherited diabetes and deafness and in pseudoxanthoma elasticum. Retina 2017;37:2008-14.

14. Rochepeau C, Kodjikian L, Garcia MA, Coulon C, Burillon C, Denis P, Delaunay B, Mathis T. Optical Coherence Tomography Angiography Quantitative Assessment of Choriocapillaris Blood Flow in Central Serous Chorioretinopathy. Am J Ophthalmol 2018;194:26-34.

15. Lang T. Twenty statistical errors even you can find in biomedical research articles. Croat Med J 2004;45:361-70.
16. Murray GD. The task of a statistical referee. Br J Surg 1988;75:664-7.

17. Okubo A, Sameshima M, Uemura A, Kanda S, Ohba N. Clinicopathological correlation of polypoidal choroidal vasculopathy revealed by ultrastructural study. Br J Ophthalmol 2002;86:1093-8.

18. Lafaut BA, Aisenbrey S, Van den Broecke C, BartzSchmidt KU, Heimann K. Polypoidal choroidal vasculopathy pattern in age-related macular degeneration: a clinicopathologic correlation. Retina 2000;20:650-4.

19. Moussa K, Bloomer MM, Schwartz DM, Pan CK, Toy BC, Crawford JB, Afshar AR. Polypoidal choroidal vasculopathy: a clinicopathologic study. Retin Cases Brief Rep 2017;11 Suppl 1:S128-31.

20. Byon I, Nassisi M, Borrelli E, Sadda SR. Impact of Slab Selection on Quantification of Choriocapillaris Flow Deficits by Optical Coherence Tomography Angiography. Am J Ophthalmol 2019;208:397-405.

21. Spaide RF, Fujimoto JG, Waheed NK. Image artifacts in Optical coherence tomography angiography. Retina 2015;35:2163-80.

22. Zhang Q, Zheng F, Motulsky EH, Gregori G, Chu Z, Chen CL, Li C, de Sisternes L, Durbin M, Rosenfeld PJ, Wang RK. A Novel Strategy for Quantifying Choriocapillaris Flow Voids Using Swept-Source OCT Angiography. Invest Ophthalmol Vis Sci 2018;59:203-11.

23. Phansalkar N, More S, Sabale A, Joshi M. Adaptive local thresholding for detection of nuclei in diversity stained cytology images. 2011 International Conference on Communications and Signal Processing. 10-12 Feb. 2011; Kerala, India. IEEE, 2011:218-20.

24. Soille P, Vincent L. Determining watersheds in digital pictures via flooding simulations. Proc. SPIE 1360, Visual Communications and Image Processing '90: Fifth in a Series, 1990.

25. Spaide RF. Choriocapillaris Flow Features Follow a Power Law Distribution: Implications for Characterization and Mechanisms of Disease Progression. Am J Ophthalmol 2016;170:58-67.

26. Borrelli E, Uji A, Sarraf D, Sadda SR. Alterations in the Choriocapillaris in Intermediate Age-Related Macular Degeneration. Invest Ophthalmol Vis Sci 2017;58:4792-8.

27. Zheng F, Zhang Q, Shi Y, Russell JF, Motulsky EH, Banta JT, Chu Z, Zhou H, Patel NA, de Sisternes L, Durbin MK, Feuer W, Gregori G, Wang R, Rosenfeld PJ. Agedependent Changes in the Macular Choriocapillaris of Normal Eyes Imaged With Swept-Source Optical Coherence Tomography Angiography. Am J Ophthalmol 
2019;200:110-22.

28. Nesper PL, Soetikno BT, Fawzi AA. Choriocapillaris Nonperfusion is Associated With Poor Visual Acuity in Eyes With Reticular Pseudodrusen. Am J Ophthalmol 2017;174:42-55.

29. Zhang Q, Shi Y, Zhou H, Gregori G, Chu Z, Zheng F, Motulsky EH, de Sisternes L, Durbin M, Rosenfeld PJ, Wang RK. Accurate estimation of choriocapillaris flow deficits beyond normal intercapillary spacing with swept source OCT angiography. Quant Imaging Med Surg 2018;8:658-66.

30. Ferrara N. Vascular endothelial growth factor: basic science and clinical progress. Endocr Rev 2004;25:581-611.

31. Takahashi H, Shibuya M. The vascular endothelial growth factor (VEGF)/VEGF receptor system and its role under physiological and pathological conditions. Clin Sci (Lond) 2005;109:227-41.

32. De Falco $\mathrm{S}$. The discovery of placenta growth factor and its biological activity. Exp Mol Med 2012;44:1-9.

Cite this article as: $\mathrm{Wu} \mathrm{H}$, Sekiryu T, Sugano Y, Itagaki K, Kasai A, Shintake H. A modified measuring method to investigate the choriocapillaris flow void of polypoidal choroidal vasculopathy with swept source optical coherence tomography angiography. Quant Imaging Med Surg 2021;11(7):3146-3156. doi: 10.21037 /qims-20-1027
33. Sasahara M, Tsujikawa A, Musashi K, Gotoh N, Otani A, Mandai M, Yoshimura N. Polypoidal choroidal vasculopathy with choroidal vascular hyperpermeability. Am J Ophthalmol 2006;142:601-7.

34. Kim K, Kim JM, Kim DG, Yu SY, Kim ES. Five-Year Follow-Up of Unaffected Fellow Eyes in Patients with Polypoidal Choroidal Vasculopathy. Ophthalmologica 2020;243:172-7.

35. Koizumi H, Kano M, Yamamoto A, Saito M, Maruko I, Sekiryu T, Okada AA, Iida T. Subfoveal Choroidal Thickness during Aflibercept Therapy for Neovascular Age-Related Macular Degeneration: Twelve-Month Results. Ophthalmology 2016;123:617-24.

36. Wong TY, Ogura Y, Lee WK, Iida T, Chen SJ, Mitchell P, Gemmy Cheung CM, Zhang Z, Leal S, Ishibashi T, Investigators P. Efficacy and Safety of Intravitreal Aflibercept for Polypoidal Choroidal Vasculopathy: TwoYear Results of the Aflibercept in Polypoidal Choroidal Vasculopathy Study. Am J Ophthalmol 2019;204:80-9. 